An anal yt i cal and numeri cal study of coupl ed transi ent nat ural convect i on and sol i di fi cat i on i n a rectangul ar encl osure

\begin{tabular}{|l|l|}
\hline 著者 & Vynnycky M, K mur a Shi geo \\
\hline $\begin{array}{l}\text { j ournal or } \\
\text { publ i cat i on t i t I e }\end{array}$ & $\begin{array}{l}\text { I nt er nat i onal Jour nal of Heat and Mass } \\
\text { Transf er }\end{array}$ \\
\hline vol une & 50 \\
\hline number & $25-26$ \\
\hline page r ange & $5204-5214$ \\
\hline year & 2007- 12-01 \\
\hline URL & ht t p: //hdl . handl e. net /2297/7662 \\
\hline
\end{tabular}




\title{
An analytical and numerical study of coupled transient natural convection and solidification in a rectangular enclosure
}

\author{
M. Vynnycky, S. Kimura* \\ Institute of Nature and Environmental Technology, Kanazawa University, \\ Kakuma-machi, Kanazawa, 920-1192, Japan. \\ ${ }^{*}$ Corresponding author. E-mail address: skimura@t.kanazawa-u.ac.jp (S. Kimura)
}

November 26, 2006

\begin{abstract}
The transient process of the solidification of a pure liquid phase-change material in the presence of natural convection in a rectangular enclosure is considered both analytically and numerically. One vertical boundary is held at a temperature below the melting point of the material, the other above; the horizontal boundaries are both assumed adiabatic. A nondimensional analysis of the problem, principally in terms of the Rayleigh $(R a)$ and Stefan $(S t)$ numbers, indicates that some asymptotic simplification is possible for materials often considered in the literature (water, gallium, lauric acid). This observation suggests a way to simplify the full problem when $R a \gg 1$ and $S t \ll 1$, giving a conventional boundary value problem for the liquid phase and pointwise-in-space first-order ODEs for the evolution in time of the solidification front. The method is tested against full $2 \mathrm{D}$ finite-element-based transient numerical simulations of solidification. In addition, simpler approaches for determining the average thickness of the solid layer, based on boundary-layer and enclosure flow correlations, are also investigated.
\end{abstract}

Keywords: solidification, natural convection, finite-element methods, asymptotics 


\section{NOMENCLATURE}

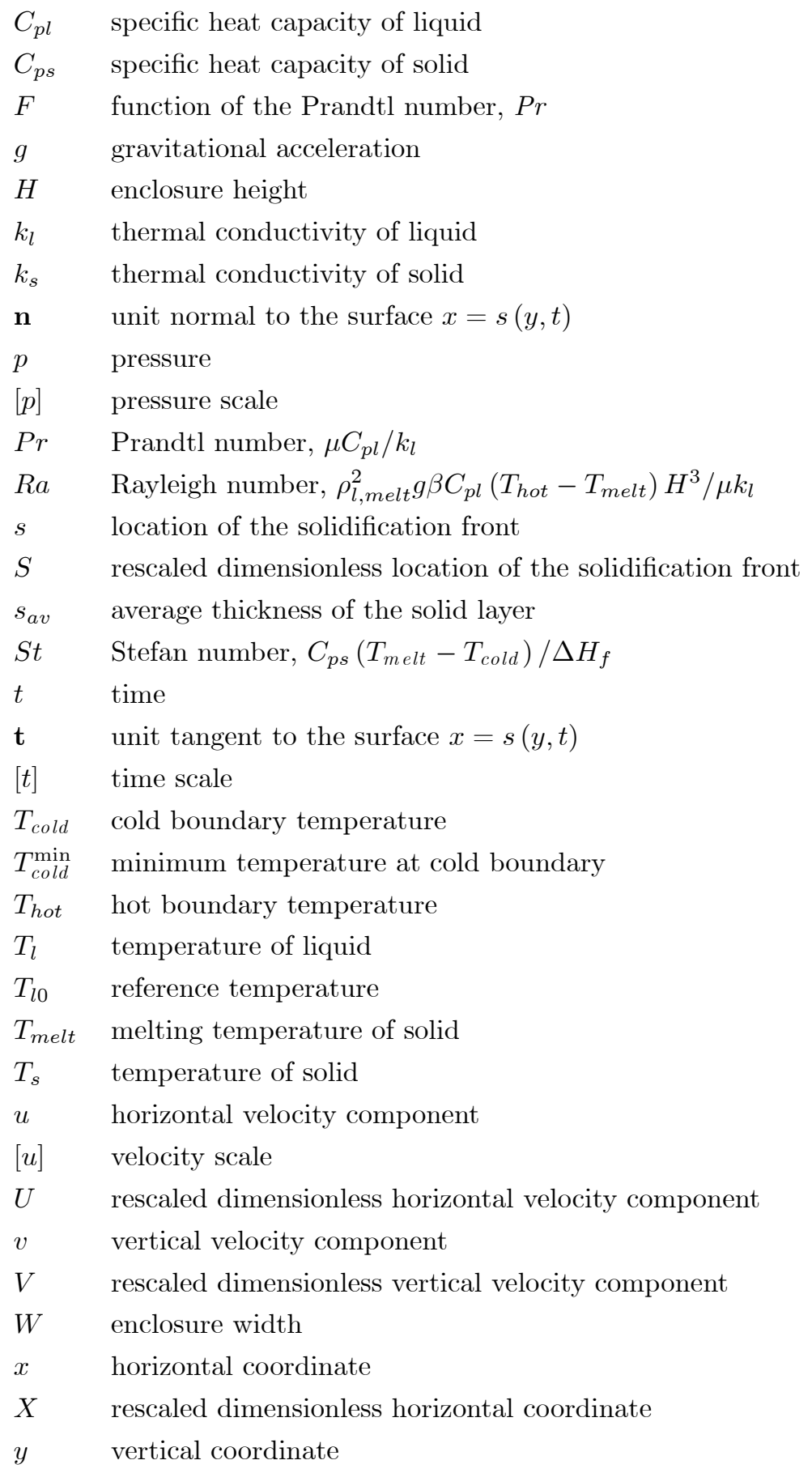


Greek

$\beta \quad$ volumetric thermal expansion coefficient

$\Gamma \quad$ dimensionless function of $y$

$\Delta H_{f} \quad$ latent heat of fusion

$\Delta \theta \quad$ increment in dimensionless temperatures $\left(\theta_{l}\right.$ and $\left.\theta_{s}\right)$

$\Delta \psi \quad$ increment in stream function, $\psi$

$\theta_{\text {cold }}$ dimensionless cold plate temperature

$\theta_{l} \quad$ dimensionless temperature of liquid

$\theta_{s} \quad$ dimensionless temperature of solid

$\kappa \quad$ dimensionless coefficient, $\frac{k_{l}\left(T_{\text {hot }}-T_{\text {melt }}\right)}{k_{s}\left(T_{m e l t}-T_{\text {cold }}\right)}$

$\kappa_{l} \quad$ liquid thermal diffusivity, $k_{l} / \rho_{l, m e l t} C_{p l}$

$\kappa_{s} \quad$ solid thermal diffusivity, $k_{s} / \rho_{s} C_{p s}$

$\lambda \quad$ enclosure aspect ratio, $W / H$

$\Lambda \quad$ dimensionless coefficient, $S t\left(\frac{k_{s}}{k_{l}}\right)\left(\frac{C_{p l}}{C_{p s}}\right)\left(\frac{\rho_{l, m e l t}}{\rho_{s}}\right)$

$\mu \quad$ liquid molecular viscosity

$\rho_{l} \quad$ liquid density

$\rho_{l, \text { melt }}$ liquid density at melting temperature

$\rho_{s} \quad$ solid density

$\varrho \quad$ dimensionless coefficient, $\rho_{s} / \rho_{l, \text { melt }}$

$\tau \quad$ dimensionless time $(\tau=t / \Lambda[t])$

$\phi \quad$ solution to transcendental equation (54)

$\varphi \quad$ phase lag

$\psi \quad$ dimensionless stream function

$\omega \quad$ cooling oscillation frequency 


\section{Introduction}

Buoyancy-driven flows with coupled solid-liquid phase change occur in a broad range of scientific and engineering fields; often cited examples are those in the solidification and melting phenomena encountered in metallurgical processes, latent heat thermal energy storage, oceanography, food processing and nuclear reactor safety.

A geometrical configuration of particular interest for such flows, owing to its simplicity and practical importance, is a rectangular enclosure in which the cooling occurs at one of the vertical walls, whilst the horizontal walls are adiabatic. This geometry has been considered for the freezing of water [1-7], the melting of tin [8] and the solidification of gallium [9-11], as well as in metal casting [12]. Recent years have also seen an increased focus on the development of numerical methods used to solve such problems [7,13,14].

The focus of this work differs from that of earlier ones by combining asymptotic analysis with numerical computations to give an improved understanding of the evolution in time of the phase-change front in solidification problems in rectangular enclosures, as well as to provide useful engineering correlations for the thickness of the solidified layer as a function of time. To illustrate this, numerical computations are carried out based around the thermophysical properties of lauric acid, $\mathrm{CH}_{3}\left(\mathrm{CH}_{2}\right)_{10} \mathrm{COOH}$, which is often used in laboratory investigations of melting-point depression and has been the subject of a couple of recent experimental and numerical studies $[7,16]$. One of the key results of the present paper is that, for substances such as water, gallium and lauric acid, the full transient 2D coupled solidification/natural convection problem can be systematically decoupled to give a conventional boundary value problem for the liquid and pointwise-in-space first-order ODEs for the evolution in time of the solidification front; furthermore, this can be used to understand how a front will move if subjected to periodic cooling. In addition, the numerical method used here is also novel: we use the arbitrary Lagrangian Eulerian formulation within commercially-available finite element software, Comsol Multiphysics [15], an approach well-suited to problems where there is isothermal phase change.

The layout of the paper is as follows. In section 2, we formulate the problem mathematically. In section 3 , it is rewritten in nondimensionalised variables, and subsequently analyzed in section 4 . Section 5 gives a description of the numerical method used. The results are presented and discussed in Section 6, and conclusions are drawn in Section 7. 


\section{Mathematical formulation}

We consider, as shown in Fig. 1, a rectangular enclosure of width $W$ and height

$H$ that initially contains liquid at temperature $T_{h o t}$, which subsequently starts to solidify when the temperature at $x=0$ is reduced to $T_{\text {cold }}$, where $T_{\text {cold }} \leq T_{\text {melt }}$, the melting temperature of the solid material; throughout, the wall at $x=W$ is held at temperature $T_{h o t}$, whereas the horizontal walls at $y=0, H$ are adiabatic. With time, a natural convection flow pattern is expected to develop, as is a solid layer; the location of the solid-liquid interface is given by $x=s(y, t)$.

\subsection{Governing equations}

For the solid region, $0 \leq x \leq s(y, t)$, we have

$$
\rho_{s} C_{p s} \frac{\partial T_{s}}{\partial t}=k_{s}\left(\frac{\partial^{2} T_{s}}{\partial x^{2}}+\frac{\partial^{2} T_{s}}{\partial y^{2}}\right)
$$

i.e. the equation for transient heat conduction. For the liquid region, $s(y, t)<$ $x<W$,we have, on using the Boussinesq approximation in the equations for transient mass, momentum and heat transfer,

$$
\begin{aligned}
\frac{\partial u}{\partial x}+\frac{\partial v}{\partial y} & =0 \\
\rho_{l, m e l t}\left(\frac{\partial u}{\partial t}+u \frac{\partial u}{\partial x}+v \frac{\partial u}{\partial y}\right) & =-\frac{\partial p}{\partial x}+\mu\left(\frac{\partial^{2} u}{\partial x^{2}}+\frac{\partial^{2} u}{\partial y^{2}}\right), \\
\rho_{l, m e l t}\left(\frac{\partial v}{\partial t}+u \frac{\partial v}{\partial x}+v \frac{\partial v}{\partial y}\right) & =-\frac{\partial p}{\partial y}+\mu\left(\frac{\partial^{2} v}{\partial x^{2}}+\frac{\partial^{2} v}{\partial y^{2}}\right) \\
\rho_{l, m e l t} C_{p l}\left(\frac{\partial T_{l}}{\partial t}+u \frac{\partial T_{l}}{\partial x}+v \frac{\partial T_{l}}{\partial y}\right) & =k_{l}\left(\frac{\partial^{2} T_{l}}{\partial x^{2}}+\frac{\partial^{2} T_{l}}{\partial y^{2}}\right) .
\end{aligned}
$$

In equation (4), for the liquid density, $\rho_{l}$, we have used for the liquid density, $\rho_{l}$, the expression

$$
\rho_{l}=\rho_{l, m e l t}\left(1-\beta\left(T-T_{\text {melt }}\right)\right) .
$$

\subsection{Boundary and initial conditions}

At $x=0$,

$$
T_{s}=T_{\text {cold }} \text { for } 0 \leq y \leq H \text {; }
$$


at $x=W$,

$$
T_{l}=T_{h o t}, u=v=0 \text { for } 0 \leq y \leq H
$$

at $y=0$,

$$
\begin{aligned}
& \frac{\partial T_{l}}{\partial y}=0, u=v=0 \text { for } s(0, t) \leq x \leq W \\
& \frac{\partial T_{s}}{\partial y}=0 \text { for } 0 \leq x \leq s(0, t)
\end{aligned}
$$

at $y=H$,

$$
\begin{aligned}
& \frac{\partial T_{l}}{\partial y}=0, u=v=0 \text { for } s(H, t) \leq x \leq W, \\
& \frac{\partial T_{s}}{\partial y}=0 \text { for } 0 \leq x \leq s(H, t) .
\end{aligned}
$$

At $x=s(y, t)$,

$$
\begin{aligned}
T_{s} & =T_{m e l t}, T_{l}=T_{m e l t}, \\
k_{s} \nabla T_{s} \cdot \mathbf{n}-k_{l} \nabla T_{l} \cdot \mathbf{n} & =\rho_{s}\left(\Delta H_{f}\right) \frac{\partial s}{\partial t} \\
(u, v) \cdot \mathbf{t} & =0, \rho_{l}\left[\frac{\partial s}{\partial t}-(u, v) \cdot \mathbf{n}\right]=\rho_{s} \frac{\partial s}{\partial t} .
\end{aligned}
$$

Here, $\mathbf{n}$ and $\mathbf{t}$ are, respectively, the unit vectors normal and perpendicular to the curve $x=s(y, t)$.

The initial conditions at $t=0$ are

$$
\begin{aligned}
T_{l}(x, y, 0) & =T_{h o t}, \\
s(y, 0) & =0 .
\end{aligned}
$$

\section{Nondimensionalisation}

We nondimensionalise with

$$
\begin{aligned}
\tilde{x} & =\frac{x}{H}, \tilde{y}=\frac{y}{H}, \tilde{s}=\frac{s}{H}, \tilde{t}=\frac{t}{[t]}, \\
\theta_{s} & =\frac{T_{s}-T_{\text {cold }}}{T_{\text {melt }}-T_{\text {cold }}}, \theta_{l}=\frac{T_{l}-T_{\text {melt }}}{T_{\text {hot }}-T_{\text {melt }}}, \\
\tilde{u} & =\frac{u}{[u]}, \tilde{v}=\frac{v}{[u]}, \tilde{p}=\frac{p}{[p]},
\end{aligned}
$$


Suitable choices for the time scale $[t]$, the velocity scale $[u]$ and the pressure scale $[p]$ are

$$
[t]=\frac{\rho_{s}\left(\Delta H_{f}\right) H^{2}}{k_{s}\left(T_{m e l t}-T_{\text {cold }}\right)},[u]=\frac{k_{l}}{H \rho_{l, \max } C_{p l}},[p]=\frac{\mu k_{l}}{H^{2} \rho_{l, \max } C_{p l}} .
$$

\subsection{Governing equations}

Equations (1)-(5) become, on dropping the tildes,

$$
\begin{aligned}
& S t \frac{\partial \theta_{s}}{\partial t}=\left(\frac{\partial^{2} \theta_{s}}{\partial x^{2}}+\frac{\partial^{2} \theta_{s}}{\partial y^{2}}\right) \\
& \frac{\partial u}{\partial x}+\frac{\partial v}{\partial y}=0 \\
& \frac{\Lambda}{\operatorname{Pr}} \frac{\partial u}{\partial t}+\frac{1}{\operatorname{Pr}}\left(u \frac{\partial u}{\partial x}+v \frac{\partial u}{\partial y}\right)=-\frac{\partial p}{\partial x}+\frac{\partial^{2} u}{\partial x^{2}}+\frac{\partial^{2} u}{\partial y^{2}}, \\
& \frac{\Lambda}{\operatorname{Pr}} \frac{\partial v}{\partial t}+\frac{1}{\operatorname{Pr}}\left(u \frac{\partial v}{\partial x}+v \frac{\partial v}{\partial y}\right)=-\frac{\partial p}{\partial y}+\frac{\partial^{2} v}{\partial x^{2}}+\frac{\partial^{2} v}{\partial y^{2}}+R a \theta_{l}, \\
& \Lambda \frac{\partial \theta_{l}}{\partial t}+u \frac{\partial \theta_{l}}{\partial x}+v \frac{\partial \theta_{l}}{\partial y}=\frac{\partial^{2} \theta_{l}}{\partial x^{2}}+\frac{\partial^{2} \theta_{l}}{\partial y^{2}} .
\end{aligned}
$$

where the Rayleigh number, $R a$, the Prandtl number, $P r$, and the Stefan number, $S t$, are given, respectively, by

$$
R a=\frac{\rho_{l, m e l t}^{2} \beta g C_{p l}\left(T_{h o t}-T_{m e l t}\right) H^{3}}{\mu k_{l}}, \operatorname{Pr}=\frac{\mu C_{p l}}{k_{l}}, S t=\frac{C_{p s}\left(T_{m e l t}-T_{\text {cold }}\right)}{\Delta H_{f}},
$$

and

$$
\Lambda=S t\left(\frac{\kappa_{s}}{\kappa_{l}}\right),
$$

where the solid and liquid thermal diffusivities, $\kappa_{s}$ and $\kappa_{l}$, are given respectively by

$$
\kappa_{s}=k_{s} / \rho_{s} C_{p s}, \kappa_{l}=k_{l} / \rho_{l, m e l t} C_{p l}
$$

\subsection{Boundary conditions}

At $x=0$,

$$
\theta_{s}=0 \text { for } 0 \leq y \leq 1
$$


at $x=\lambda$, where $\lambda=W / H$,

$$
\theta_{l}=1, u=v=0 \text { for } 0 \leq y \leq 1
$$

at $y=0$,

$$
\begin{aligned}
& \frac{\partial \theta_{l}}{\partial y}=0, u=v=0 \text { for } s(0, t) \leq x \leq \lambda \\
& \frac{\partial \theta_{s}}{\partial y}=0 \text { for } 0 \leq x \leq s(0, t)
\end{aligned}
$$

at $y=1$,

$$
\begin{aligned}
& \frac{\partial \theta_{l}}{\partial y}=0, u=v=0 \text { for } s(0, t) \leq x \leq \lambda \\
& \frac{\partial \theta_{s}}{\partial y}=0 \text { for } 0 \leq x \leq s(0, t) .
\end{aligned}
$$

At $x=s(y, t)$,

$$
\begin{aligned}
\theta_{s} & =1, \theta_{l}=0 \\
\nabla \theta_{s} \cdot \mathbf{n}-\kappa \nabla \theta_{l} \cdot \mathbf{n} & =\frac{\partial s}{\partial t}, \\
(u, v) \cdot \mathbf{t} & =0 \\
(u, v) \cdot \mathbf{n} & =\Lambda(1-\varrho) \frac{\partial s}{\partial t},
\end{aligned}
$$

where

$$
\kappa=\frac{k_{l}\left(T_{\text {hot }}-T_{\text {melt }}\right)}{k_{s}\left(T_{\text {melt }}-T_{\text {cold }}\right)}, \varrho=\frac{\rho_{s}}{\rho_{l, \text { melt }}} .
$$

The initial conditions at $t=0$ are

$$
\begin{aligned}
\theta_{l}(x, y, 0) & =1, \\
s(y, 0) & =0 .
\end{aligned}
$$

\section{Analysis}

We have six dimensionless parameters:

$$
R a, S t, \kappa, \operatorname{Pr}, \varrho, \Lambda \text {. }
$$


To fix ideas, we focus on processes such as the freezing of water, the solidification of gallium and the solidification of lauric acid, the values of the relevant physical parameters for which are given in Table 1. From these, and with

$$
\left(T_{\text {melt }}-T_{\text {cold }}\right),\left(T_{\text {hot }}-T_{\text {melt }}\right) \sim 5 \mathrm{~K}, H \sim 0.5 \mathrm{~m},
$$

we have

$$
R a \gg 1, \kappa \sim 1, \varrho \sim 1,
$$

whereas characteristic values for the other three parameters are given in Table 2. For all three cases, $S t \ll 1$, which suggests that (18) can be reduced to

$$
\frac{\partial^{2} \theta_{s}}{\partial x^{2}}+\frac{\partial^{2} \theta_{s}}{\partial y^{2}}=0
$$

at leading order in St. Note incidentally that for the solidification of metals other than gallium, e.g. copper, tin [8], St will be $O(1)$ or higher for the temperature differences given above, since the value of $C_{p s} / \Delta H_{f}$ is considerably higher than that for gallium. Thus, the analysis given below mat hold for such metals, but only if the temperature differences are fractions of a degree.

Next, several levels of decoupling are possible, depending on the value of $\Lambda$.

\section{$4.1 \Lambda \ll 1$}

In this case, equations (20)-(22) become, at leading order,

$$
\begin{aligned}
& \frac{1}{\operatorname{Pr}}\left(u \frac{\partial u}{\partial x}+v \frac{\partial u}{\partial y}\right)=-\frac{\partial p}{\partial x}+\frac{\partial^{2} u}{\partial x^{2}}+\frac{\partial^{2} u}{\partial y^{2}}, \\
& \frac{1}{P r}\left(u \frac{\partial v}{\partial x}+v \frac{\partial v}{\partial y}\right)=-\frac{\partial p}{\partial y}+\frac{\partial^{2} v}{\partial x^{2}}+\frac{\partial^{2} v}{\partial y^{2}}+R a \theta_{l}, \\
& u \frac{\partial \theta_{l}}{\partial x}+v \frac{\partial \theta_{l}}{\partial y}=\frac{\partial^{2} \theta_{l}}{\partial x^{2}}+\frac{\partial^{2} \theta_{l}}{\partial y^{2}},
\end{aligned}
$$

whereas (32) reduces to

$$
(u, v) \cdot \mathbf{n}=0
$$

Thus, the only time-dependence left in the problem occurs via (30). Furthermore, the fact that $\nabla \theta_{l} \cdot \mathbf{n} \sim R a^{\frac{1}{4}}$ in (30) in the liquid at the interface, as is the case in the case of natural convection in the absence of solidification, suggests that the thickness of the solid layer will be much less than the height of the layer. A consistent asymptotic structure for the solution is then obtained by 
writing, for the solid region,

$$
x=R a^{-\frac{1}{4}} X, s=R a^{-\frac{1}{4}} S, t=R a^{-\frac{1}{2}} \hat{t},
$$

so that equation (18) reduces to just

$$
\frac{\partial^{2} \theta_{s}}{\partial X^{2}}=0
$$

Using equations (23) and (29), we obtain

$$
\theta_{s}=\frac{X}{S(y, \hat{t})}
$$

For the liquid, equations (19)-(22) will reduce, in the vicinity of $X=S(y, \tau)$, at leading order, to the steady-state boundary-layer equations, written in terms of coordinates that are locally normal and tangential to the solidification front and subject to boundary conditions (29)-(31). Also, as we are assuming that the solid layer is thin, boundary conditions can be effectively taken at $X=0$. In addition, we reduce equation (31) to

$$
U=0, V=0,
$$

although we note that, as is well known from the classical Stefan solution for 1D solidification, initially $S(\hat{t}) \sim \hat{t}^{\frac{1}{2}}$, so that equation (31) will be strictly speaking only valid once $\hat{t} \gg(1-\varrho)^{2} \Lambda^{2}$.

Further, these considerations imply the solution for the liquid is, at leading order, can be treated as being the same as that for steady state natural convection in a rectangular cavity. Whilst this would still need to be computed numerically, it is a considerably simpler computational task than to solve the full time-dependent moving boundary problem. Once such a computation is carried out, we will have the evolution equation for $s$,

$$
\frac{\partial s}{\partial t}=\frac{1}{s}-\kappa\left(\frac{\partial \theta_{l}}{\partial x}\right)_{x=0},
$$

subject to the initial condition

$$
s(y, 0)=0 .
$$

Here, $\left(\frac{\partial \theta_{l}}{\partial x}\right)_{x=0}$ is the temperature derivative computed for a steady-state prob- 
lem in an enclosure without solidification, and hence is a function of $y$ only. This can be integrated with respect to $t$ to give $s$ in implicit form as

$$
\Gamma^{-1} \log (1-\Gamma s)+s=-\Gamma t
$$

where $\Gamma=\kappa\left(\frac{\partial \theta_{l}}{\partial x}\right)_{x=0}$. This suggests that, for small times,

$$
s \sim t^{\frac{1}{2}}
$$

as one would expect from the Stefan solution; note also that this is independent of $\Gamma$, which therefore indicates one-dimensional solidification. For large times,

$$
s \sim \frac{1}{\Gamma}\left(1-\exp \left(-\Gamma^{2} t\right)\right)
$$

we return to this equation later.

Also of interest is whether $\mathrm{Pr}$-dependent Nusselt number correlation, $F(\mathrm{Pr})$, based on a similarity solution to the boundary-layer equations for steady state natural convection past a vertical surface, can be of use in determining the average solid thickness. To see this, we argue as follows. In a rectangular cavity, the average temperature at the outer edge of the boundary layer at the melting front will be $\left(T_{\text {melt }}+T_{h o t}\right) / 2$. Therefore, we consider a vertical boundary at temperature $T_{\text {melt }}$ adjacent to fluid at temperature $\left(T_{\text {melt }}+T_{\text {hot }}\right) / 2$; for $\left(\frac{\partial \theta_{l}}{\partial x}\right)_{x=0}$ in (39), we use the value that can be extracted from the correlation given by, amongst others, Bejan [17]:

$$
F(P r)=0.503\left(\frac{P r}{P r+0.986 \operatorname{Pr}^{\frac{1}{2}}+0.492}\right)^{\frac{1}{4}} .
$$

This gives

$$
\frac{\partial s}{\partial t}=\frac{1}{s}-\frac{\kappa}{2}\left(\frac{R a}{2}\right)^{\frac{1}{4}}(1-y)^{-\frac{1}{4}} F(P r),
$$

which can then be solved for $s$, and then the average solid thickness, $s_{a v}$, defined by

$$
s_{a v}:=\int_{0}^{1} s(y, t) d y,
$$

can be found; in particular, at steady state, this would give

$$
s_{a v} \rightarrow \frac{8\left(\frac{R a}{2}\right)^{-\frac{1}{4}}}{5 \kappa F(\operatorname{Pr})}, \text { as } t \rightarrow \infty .
$$


An alternative approach would be to balance average heat fluxes at the solidification front by writing

$$
\frac{d s_{a v}}{d t}=\frac{1}{s_{a v}}-\frac{2}{3} \kappa\left(\frac{R a}{2}\right)^{\frac{1}{4}} F(P r)
$$

leading to

$$
s_{a v} \rightarrow \frac{3\left(\frac{R a}{2}\right)^{-\frac{1}{4}}}{2 \kappa F(\operatorname{Pr})}, \text { as } t \rightarrow \infty .
$$

However, neither (44) nor (46) take into account the aspect ratio of the enclosure. This can be done by using instead the Berkovsky-Polevikov correlations recommended by Catton [18]. Using these, we would have the following evolution equations for $s_{a v}$ :

$$
\frac{d s_{a v}}{d t}=\frac{1}{s_{a v}}-0.18 \kappa\left(\frac{R a P r}{0.2+P r}\right)^{0.29}\left(\lambda\left(1-s_{a v}\right)\right)^{-0.13},
$$

for $1<\frac{1}{\lambda\left(1-s_{a v}\right)}<2,10^{-3}<\operatorname{Pr}<10^{5}, 10^{3}<\left(\frac{P r}{0.2+P r}\right) R a\left(\lambda\left(1-s_{a v}\right)\right)^{3}$;

$$
\frac{d s_{a v}}{d t}=\frac{1}{s_{a v}}-0.22 \kappa\left(\frac{R a P r}{0.2+P r}\right)^{0.28}\left(\lambda\left(1-s_{a v}\right)\right)^{0.09}
$$

for $2<\frac{1}{\lambda\left(1-s_{a v}\right)}<10, \operatorname{Pr}<10^{5}, 10^{3}<R a^{13}$. We will compare the results from equations (44), (46) and (48) in Section 6.

\section{$4.2 \Lambda \sim 1$}

In this case, the flow in the liquid will evolve with time. However, the solid layer will not become thicker than its steady state value, which suggests that the scaling given in (38) will still hold. Consequently, the problem can still be decoupled by solving for the natural convective flow in a rectangular cavity, except that $\left(\frac{\partial \theta_{l}}{\partial x}\right)_{x=0}$ will now be time-dependent. Although the analytical solution (41) is not now valid, equation (39) still will be, and the complete problem can be solved by first solving for the velocity and temperature fields in the fluid and then the first-order ODE in (39). 


\subsection{Time-dependent cooling}

In addition, a generalization of equation (39), that is of use in problems when it is desired to control the movement of the solidification front, is possible if the temperature of the cold boundary varies in space and time, so that $T_{\text {cold }}=$ $T_{\text {cold }}(y, t)$. We nondimensionalise $T_{s}$ by

$$
\theta_{s}=\frac{T_{s}-T_{\text {cold }}^{\min }}{T_{\text {melt }}-T_{\text {cold }}^{\min }}
$$

where $T_{\text {cold }}^{\min }=\min \left\{T_{\text {cold }}(y, t) \mid t \geq 0,0<y<1\right\}$, and obtain

$$
\theta_{s}=\frac{\left(1-\theta_{\text {cold }}(y, t)\right) x}{s(y, t)}+\theta_{\text {cold }}(t)
$$

giving, as the evolution equation for $s$,

$$
\frac{\partial s}{\partial t}=\frac{1-\theta_{\text {cold }}(y, t)}{s}-\kappa\left(\frac{\partial \theta_{l}}{\partial x}\right)_{x=0},
$$

subject to (40).

$$
\frac{\partial s}{\partial t}=\frac{1-\theta_{\text {cold }}(y, t)}{s}-\kappa\left(\frac{\partial \theta_{l}}{\partial x}\right)_{x=0} .
$$

Now, suppose

$$
\theta_{\text {cold }}(y, t)=\epsilon \Theta_{\text {cold }}(y, t),
$$

where $\epsilon \ll 1$ and $\Theta_{\text {cold }}$ is an $O(1)$ function. We see how this affects the location of the solidification front. Setting

$$
s=s_{0}(y, t)+\epsilon s_{1}(y, t)+O\left(\epsilon^{2}\right),
$$

we have at $O\left(\epsilon^{0}\right)$,

$$
\frac{\partial s_{0}}{\partial t}=\frac{1}{s_{0}}-\kappa\left(\frac{\partial \theta_{l}}{\partial x}\right)_{x=0}
$$

whereas as $O(\epsilon)$,

$$
\frac{\partial s_{1}}{\partial t}=-\frac{\Theta_{\text {cold }}}{s_{0}}-\frac{s_{1}}{s_{0}^{2}} .
$$

Equation (51) is of course the same as (39), but more interesting is equation (52), which indicates that convection in the melt will not contribute to this 
balance. Further, if we assume periodic heating and cooling of the form

$$
\Theta_{\text {cold }}=\sin \omega t
$$

then, after an the initial transient during which the leading order solution settles to a steady state, the governing equation for $s_{1}$ is

$$
\frac{\partial s_{1}}{\partial t}=-\frac{\sin \omega t}{s_{0}(y)}-\frac{s_{1}}{s_{0}^{2}(y)},
$$

which can be solved exactly. The large time solution for $s_{1}$ is then

$$
s_{1}(y, t)=-\frac{1}{2 i s_{0}(y)}\left(\frac{\exp (i \omega t)}{\left[\frac{1}{s_{0}^{2}(y)}+i \omega\right]}-\frac{\exp (-i \omega t)}{\left[\frac{1}{s_{0}^{2}(y)}-i \omega\right]}\right)
$$

which can be further rearranged to give

$$
s_{1}(y, t)=\frac{s_{0}(y) \sin (\omega t-\varphi+\pi)}{\left[1+\omega^{2} s_{0}^{4}(y)\right]^{\frac{1}{2}}}
$$

where

$$
\sin \varphi=\frac{\omega s_{0}^{2}(y)}{\left[1+\omega^{2} s_{0}^{4}(y)\right]^{\frac{1}{2}}}, \quad \cos \varphi=\frac{1}{\left[1+\omega^{2} s_{0}^{4}(y)\right]^{\frac{1}{2}}},
$$

i.e.

$$
\tan \varphi=\omega s_{0}^{2}(y)
$$

Consequently, we see that the location of the front oscillates in a complex manner, with an amplitude, $\left[1+\omega^{2} s_{0}^{4}(y)\right]^{-\frac{1}{2}} s_{0}(y)$, and phase lag, $\pi-\varphi$, that depend both on the leading order $y$-position of the front, $s_{0}(y)$, and the frequency, $\omega$ Whilst the focus of the numerical work in this paper is basically to find $s_{0}$, future work will focus on determining numerically the accuracy of the expression for $s_{1}$.

\section{Numerical implementation}

The full problem, involving equations (18)-(22) subject to boundary conditions (23)-(31) and initial conditions (33) and (34), was solved numerically using the finite element-based PDE software, Comsol Multiphysics [15]. All computations were performed on a Dell Optiplex GX520 computer with a $3 \mathrm{GHz}$ processor 
and 1 GB RAM and required no more half an hour of CPU time.

First, a grid independence study was carried out on the problem without solidification. Lagrangian P2-P1 quadrilateral elements and second-order quadrilateral elements for the Navier Stokes and the heat equations, respectively, were used on three different mapped meshes, having around 800, 1,400 and 3,400 elements and corresponding to 11,000, 20,000 and 45,000 degrees of freedom, respectively; the results of this are given in Fig. 2. The difference between the meshes lies only in the number of points used to discretize the boundary layers; 5,10 and 20 points, respectively, are used within a dimensionless distance of 0.05 from each boundary. For all cases, the same convergence criterion, namely

$$
\left(\frac{1}{N_{d o f}} \sum_{i=1}^{N_{\text {dof }}}\left|E_{i}\right|^{2}\right)^{\frac{1}{2}}<\epsilon
$$

was applied; here $N_{d o f}$ is the number of degrees of freedom, $E_{i}$ is the estimated error in the current approximation to the $i$ th component of the true solution vector and $\epsilon=10^{-6}$. Lower values of $\epsilon$ were also tried, but the results were practically indistinguishable. The results of the mesh independence study are given in Fig. 2, which compares the value of $\partial \theta_{l} / \partial x$ at $x=0$ for $R a=10^{7}$. We see that an approximate doubling of the number of mesh elements, in going from 1,400 to 3,400 leads to an almost indiscernible difference in the local values of $\partial \theta_{l} / \partial x$. In view of this, and the fact more degrees of freedom are necessary for the problem with solidification, the mesh having 1,400 elements was judged to be appropriate for the computations. Results from computations were required as input to equation (39) for the asymptotic approach.

For the problem with solidification, both steady-state and transient computations were performed. Both types require the use of Comsol Multiphysics' Deformed Mesh mode, whereby an arbitrary Lagrangian-Eulerian formulation is used in order to solve free or moving boundary problems. For the steady-state computations, a pure conduction problem was solved first, and the solution for this was used as input for the software's parametric non-linear solver to find converged solutions for increasing $R a$ values. Specific details concerning the solver can be found in the software manual [15]; here, we point out that Newton iteration is used for solving the nonlinear equation system that arises in the steady-state case, whereas a method of lines discretization is used for the time-dependent case, and that the solver is an implicit time-stepping scheme 
which uses variable order variable-stepsize backward differentiation formulae. Note also that when solving the full problem with solidification, the number of degrees of freedom is somewhat greater than that indicated earlier. This is because the temperature in the solid region and the $2 \mathrm{D}$ mesh displacements also have to be solved for; for example, it was found that computer memory problems were encountered even for a mesh having only 1400 elements in the liquid region, particularly for higher values of $R a$. Consequently, it proved possible to obtain solutions to the full solidification problem for $R a$ as high as order $10^{7}$ by using a mesh with around 800 elements in the liquid.

As usual, a major difficulty for the transient computations is the fact that the solid region initially has zero thickness. To overcome this problem, we commenced the integration by using the classical 1D Stefan solution, given by

$$
\begin{aligned}
\theta_{s} & =\frac{\operatorname{erf}\left(\frac{x}{2 \sqrt{\kappa_{s} t}}\right)}{\operatorname{erf}(\phi)} \text { if } 0<x<x_{c}(t), \\
\theta_{l} & =1-\frac{\operatorname{erfc}\left(\frac{x}{2 \sqrt{\kappa_{l} t}}\right)}{\operatorname{erfc}\left(\phi\left(\frac{\kappa_{s}}{\kappa_{l}}\right)^{\frac{1}{2}}\right)} \text { if } x_{c}(t)<x<1,
\end{aligned}
$$

where $x_{c}(t)=2 \phi \sqrt{\kappa_{s} t}$ and $\phi$ is given by the solution to the following transcendental equation:

$$
\frac{\exp \left(-\phi^{2}\right)}{\operatorname{erf}(\phi)}-\left(\frac{\kappa_{s}}{\kappa_{l}}\right)^{\frac{1}{2}} \frac{\kappa \exp \left(-\phi^{2}\left(\frac{\kappa_{s}}{\kappa_{l}}\right)\right)}{\operatorname{erfc}\left(\phi\left(\frac{\kappa_{s}}{\kappa_{l}}\right)^{\frac{1}{2}}\right)}=\frac{\pi^{\frac{1}{2}} \phi}{S t} .
$$

Using the values for the physical properties given in Table 1, we obtain $\phi=0.1156$. For the transient computations, the convergence criterion at each time step was taken as

$$
\left(\frac{1}{N_{d o f}} \sum_{i=1}^{N_{d o f}}\left(\frac{\left|E_{i}\right|}{A_{i}+R\left|U_{i}\right|}\right)^{2}\right)^{\frac{1}{2}}<1,
$$

where $\left(U_{i}\right)$ is the solution vector corresponding to the solution at a certain time step, $A_{i}$ is the absolute tolerance for the $i$ th degree of freedom, and $R$ is the relative tolerance; for the computations, $R=0.01, A_{i}=0.001$ for $i=1, . ., N_{d o f}$ were used. 


\section{Results and discussion}

As the main purpose here is to compare the results of full numerical simulation with the asymptotic approach, as well as to determine correlations between the solid thickness and the Rayleigh number, we focus here only on the case when $\lambda=1$ and $\theta_{\text {cold }}(t) \equiv 0$. Although the results are presented in terms of nondimensional parameters, they are based around the properties of lauric acid, as shown in Table 1. Also, we have chosen $T_{\text {cold }}$ and $T_{\text {hot }}$ so that $\kappa=3$. In practice, this would correspond to $T_{\text {cold }}$ and $T_{\text {hot }}$ being related by

$$
T_{\text {hot }}+3 T_{\text {cold }}=4 T_{\text {melt }} ;
$$

in turn, this gives $\Lambda=S t=0.05$. There were several reasons for choosing the thermophysical properties of lauric acid, rather than water or gallium, for this analysis:

- for practical applications, the value of $\Lambda$ for water turns out to be large enough that the assumption that $\Lambda \ll 1$ may no longer be valid;

- for water, density inversion occurs at around $4^{\circ} \mathrm{C}$, giving rise to a velocity flow field with a secondary recirculation loop $[3,5-7]$ - it is unlikely that the given boundary-layer heat-flux correlations $[17,18]$ could be valid for such a flow field;

- computation times for gallium turned out to be much lengthier, presumably because it has a much lower Prandtl number than lauric acid, which increases the non-linearity in equations (20) and (21).

First, we present some results from the full numerical simulation, before proceeding to a comparison with analysis.

Fig. 3 shows the location at steady state of the solidification front for increasing values of $R a$. For the case of conduction only, it can be shown that the front will lie at $x=(1+\kappa)^{-1}$; using the parameters in Tables 1 and 2 , we obtain that $x=0.25$, as shown in the figure. As the Rayleigh number is increased, there appears to be a regime for $R a$ as high as $10^{4}$ where the upper part of the solidification front lies closer to the cooling wall than for the case of pure conduction, but the lower part lies further away. Thereafter, as $R a$ is increased further, the whole front is shifted further to the left, as the effect of convection in the liquid phase increases. As may be expected, since the heat 
flux due to natural convection is highest at the upper part of the solidification front, that is where the front lies closest to the cooling wall.

Figs. 4(a)-4(c) show the evolution of the isotherms with time for $R a=10^{7}$; because of the way the equations were programmed in the software, it was most convenient to plot the solution at the prescribed values of $\tau$, which is related to the actual time $t$ by

$$
\tau=\frac{t}{\Lambda[t]} .
$$

The isotherms in the solid are characteristic of heat transport due to conduction, whereas in the fluid we see thermal boundary layers near the solidification front, as well as at the heated wall on the right; in between, there is vertical stratification. Although Figs. 4(b and 4(c) may look identical, there is actually a difference near the point where the solidification front meets the lower horizontal boundary. As we will see from later figures, it is this point that determines when a steady state is finally reached.

Figs. 5(a)-5(c) show the streamfunction, $\psi$, defined by

$$
u=\frac{\partial \psi}{\partial y}, v=-\frac{\partial \psi}{\partial x}
$$

show the corresponding streamlines for $R a=10^{7}$. Evident here are viscous boundary layers, particularly at the melting isotherm and the cooling wall. The flow here is in an anticlockwise direction.

Fig. 6 shows the location of the solidification front at three different times for $R a=10^{7}$, as predicted by the analytically-based method outlined in section 4 and the full numerical solution; these results are best discussed in the context of the average solid thickness, $s_{a v}$, which is shown in Fig. 7. First, we should note that the result from numerical simulation in Fig. 6 for $\tau=1$ is in fact the steady state solution, as is evident from the fact that the relevant curve in Fig. 7 reaches a plateau for this value of $\tau$. In Fig. 6, the asymptotic solution shadows the numerical solution very well for all values of $\tau$ for $y>0.1$; for $y \leq 0.1$, there is a small discrepancy at $\tau=0.01$ which becomes greater as $\tau$ increases. Furthermore, this region appears to adversely affect the prediction of the average solid layer thickness at this value of $R a$ in Fig. 7. In fact, this figure also compares the results obtained when the liquid is assumed to be at steady state $(\Lambda \ll 1)$, and when it is assumed to evolve $(\Lambda \sim 1)$. We see that if it assumed to evolve, the agreement with the full numerical solution for $s_{a v}$ is good for small values of $\tau$; however, after $\tau \approx 0.1$, the agreement is less good and the 
method overpredicts the thickness of the solid layer to the same extent as the method with $\Lambda \ll 1$. Why this discrepancy at large times occurs can be found by referring to Fig. 2 and equation (42). From Fig. 2, it is clear that $\left(\frac{\partial \theta_{l}}{\partial x}\right)_{x=0}$, and hence $\Gamma$, are smallest near the bottom of the enclosure. Consequently, not only is $s$ largest there, as one would expect from equation (42), but $s$ decays most slowly there, with $t \sim \Gamma^{-1 / 2}$ being the appropriate timescale estimate for steady state.

An important question is whether the scaling for $s$ suggested by equation (38) is actually borne out in practice. This is determined in Fig. 8, where the steady state values of $\log s_{a v}$ are plotted against $\log R a$. We see that a distinct trend emerges for values of $R a$ greater than $10^{4} ; s_{a v}$ for $R a>10^{4}$ is well approximated by

$$
s_{a v}=2.17 R a^{-0.271}
$$

the fact that the exponent differs slightly from $-1 / 4$ is reminiscent of the difference between the Nusselt number correlation for a boundary layer in semiinfinite fluid and in an enclosure. Nevertheless, the value is sufficiently close to $-1 / 4$ to suggest that the scaling for $s$ given (38) is correct.

In Fig. 9, we evaluate whether any of the alternatives given in equations (44), (46) and (48) are able to predict accurately the evolution in time of $s_{a v}$. The results shown, for $R a=10^{7}$, indicate the local boundary layer solution works best, although all solutions underpredict the actual value and indicate that the steady state occurs sooner than is predicted by the full numerical solution. Fig. 10 shows the prediction for $s_{a v}$ at steady state as a function of $R a$; for all values of $R a$, the local boundary layer solution approximates the full numerical solution best.

\section{Conclusions}

In this paper, we have considered both analytically and numerically the solidification of a phase-change material in the presence of natural convection in a rectangular enclosure. Asymptotic analysis was carried out in terms of the Rayleigh $(R a)$ and Stefan $(S t)$ numbers for the regime where $R a \gg 1$ and $S t \ll 1$. Computations were carried out using the finite-element software Comsol Multiphysics. The asymptotic analysis enables us to decouple the fluid flow and heat transfer problem in the liquid from the heat transfer problem in the

solid, and is able to describe the quantitative features of the numerical solutions 
very well for all times for about $90 \%$ of the height of the enclosures. However, complications arise near the lower part of the enclosure; it appears that, in the final $10 \%$, the analytical solution is not uniformly valid for all time, and tends to overestimate the final thickness of the solid layer. A simpler analytical approach, which balances the averaged heat flux over the length of the solidification front, tends to underestimate the final thickness of the solid layer, although is quite accurate for $R a=10^{8}$.

Although the numerical results presented here were for solidification occurring as a result of cooling at a vertical boundary held at a constant temperature, the analysis presented here can be used for interpreting solidification by means of time-dependent cooling also.

\section{Acknowledgement}

The first author acknowledges the financial support of the Japanese Society for the Promotion of Science. 


\section{References}

[1] G. de Vahl Davis, E. Leonardi, P. H. Wong, G. H. Yeoh, Natural convection in a solidifying liquid, in: R. W. Lewis and K. Morgan (Eds), Numerical Methods in Thermal Problems, Pineridge Press, Swansea, 1989, 6 (1) pp. 410-420.

[2] Z. Zhang, A. Bejan, Solidification in the presence of high Rayleigh number convection in an enclosure cooled from the side, International Journal of. Heat and Mass Transfer, 33 (4) (1990) 661-671.

[3] S. L. Braga, R. Viskanta, Effect of the water density extremum on the solidification process, in: J. F. Keffer, R. K. Shah, E. N. Ganic (Eds.), Experimental Heat Transfer, Fluid Mechanics and Thermodynamics, Elsevier, Barking, Essex and Amsterdam, 1991, pp. 1185-1192.

[4] P. H. Oosthuizen, Numerical study of the steady state freezing of water in a rectangular enclosure, in: R. W. Lewis (Ed.), Numerical Methods in Thermal Problems, Pineridge Press, Swansea, 1993, VIII (1) pp. 92-103.

[5] P. H. Oosthuizen, J. T. Paul, A numerical study of the steady state freezing of water in an open rectangular cavity, International Journal of Numerical Methods for Heat and Fluid Flow, 6(5) (1996) 3-16.

[6] M. Giangi, F. Stella, T. A. Kowalewski, Phase change problems with free convection: fixed grid numerical simulation, Computing and Visualization in Science, 2 (1999) 123-130.

[7] T. J. Scanlon, M. T. Stickland, A numerical analysis of buoyancy-driven melting and freezing, International Journal of Heat and Mass Transfer, 47 (3) (2004) 429-436.

[8] C. J. Ho, C. H. Chu, Periodic melting within a square enclosure with an oscillatory surface temperature, International Journal of. Heat and Mass Transfer, 36 (3) (1993) 725-733.

[9] C. Gau, R. Viskanta, Melting and solidification of a pure metal on a vertical wall, Journal of Heat Transfer 108 (1986) 174-181.

[10] A. D. Brent, V. R. Voller, K. J. Reid, Enthalpy-porosity technique for modelling convection-diffusion phase change: application to the melting of a pure metal, Numerical Heat Transfer, 13 (1988) 297-318. 
[11] M. Mbaye, E. Bilgen, Phase change process by natural convection-diffusion in rectangular enclosures, Heat and Mass Transfer, 37 (2001) 35-42.

[12] J. Szimmat, Numerical simulation of solidification processes in enclosures, Heat and Mass Transfer, 38 (2002) 279-293.

[13] R. T. Tenchev, J. A. Mackenzie, T. J. Scanlon, M. T. Stickland, Finite element moving mesh analysis of phase change problems with natural convection, International Journal of Heat and Fluid Flow, 26(4) (2005) 597-612.

[14] www.fluent.com.

[15] Comsol Multiphysics 3.3, http://www.comsol.com.

[16] A. Bose, D. A. Scott, B. R. Baliga, An experimental investigation of melting in the presence of natural convection, in J. P. Meyer (ed.), Proceedings of the $1^{\text {st }}$ International Conference on Heat Transfer, Fluid Dynamics and Thermodynmics, Part 2, vol. 1, Kruger National Park, Skukuza, South Africa, 8-10 April 2002, pp. 678-683.

[17] A. Bejan, Heat Transfer, John Wiley and Sons, Inc., New York, 1993, pp.

[18] I. Catton, Natural convection in enclosures, 6th Int. Heat Transfer Conf., Toronto, 1978, Vol. 6, 1979, pp. 13-43.

[19] H. S. Carslaw, J. C. Jaeger, Conduction of Heat in Solids, 2nd Edition, OUP, 1959, pp. 287-288. 


\section{FIGURE CAPTIONS}

Fig. 1: Schematic diagram of solidification in an enclosure

Fig. 2: Mesh independence study

Fig. 3: Steady state location of the solidification front for different values of $R a$

Fig. 4: Isotherms for flow at $R a=10^{7}$ at: (a) $\tau=10^{-2}$; (b) $\tau=10^{-1}$; (c) $\tau=1$

Fig. 5: Streamlines for flow at $R a=10^{7}$ at: (a) $\tau=10^{-2}(\Delta \psi=10$, with $0 \leq \psi \leq 50)$; (b) $\tau=10^{-1}(\Delta \psi=6$, with $0 \leq \psi \leq 30)$; (c) $\tau=1(\Delta \psi=5$, with $0 \leq \psi \leq 25)$

Fig. 6: Evolution of $s$ towards a steady state for $R a=10^{7}$, calculated using equation (39) and full numerical simulation, at $\tau=10^{-2}, 10^{-1}, 1$

Fig. 7: Comparison of the average solid thickness, $s_{a v}$, as a function of $\tau$ for $R a=10^{7}$

Fig. 8: $\log s_{a v}$ as a function of $\log R a$ at steady state

Fig. 9: Comparison of the time evolution of $s_{a v}$ for $R a=10^{7}$, using the full numerical solution and three different liquid-phase heat flux correlations

Fig. 10: Comparison of $\log \mathrm{s}_{a v}$ as a function of $\log R a$, using the full numerical solution and three different liquid-phase heat flux correlations 


$\begin{array}{lllll} & \text { water } & \text { gallium } & \text { lauric acid } & \text { units } \\ C_{p l} & 4180 & 381.5 & 2394 & \mathrm{Jkg}^{-1} \mathrm{~K}^{-1} \\ C_{p s} & 2217 & 381.5 & 2155 & \mathrm{Jkg}^{-1} \mathrm{~K}^{-1} \\ k_{l} & 0.578 & 32 & 0.6098 & \mathrm{Wm}^{-1} \mathrm{~K}^{-1} \\ k_{s} & 1.918 & 32 & 0.6098 & \mathrm{Wm}^{-1} \mathrm{~K}^{-1} \\ T_{m e l t} & 273 & 302.78 & 316.5 & \mathrm{~K} \\ \Delta H_{f} & 333000 & 80160 & 183000 & \mathrm{Jkg}^{-1} \\ \rho_{l 0} & 999.972 & 6093 & 869.0 & \mathrm{kgm}^{-3} \\ \rho_{s} & 918.0 & 6093 & 1005.5 & \mathrm{kgm}^{-3} \\ \mu & 0.00175 & 0.00181 & 0.0071 & \mathrm{kgm}^{-1} \mathrm{~S}^{-1}\end{array}$

Table 1

$\begin{array}{llll} & \text { water } & \text { gallium } & \text { lauric acid } \\ \text { St } & 0.02 & 0.02 & 0.015 \\ \operatorname{Pr} & 12 & 0.02 & 119 \\ \Lambda & 0.2 & 0.02 & 0.017\end{array}$

Table 2 


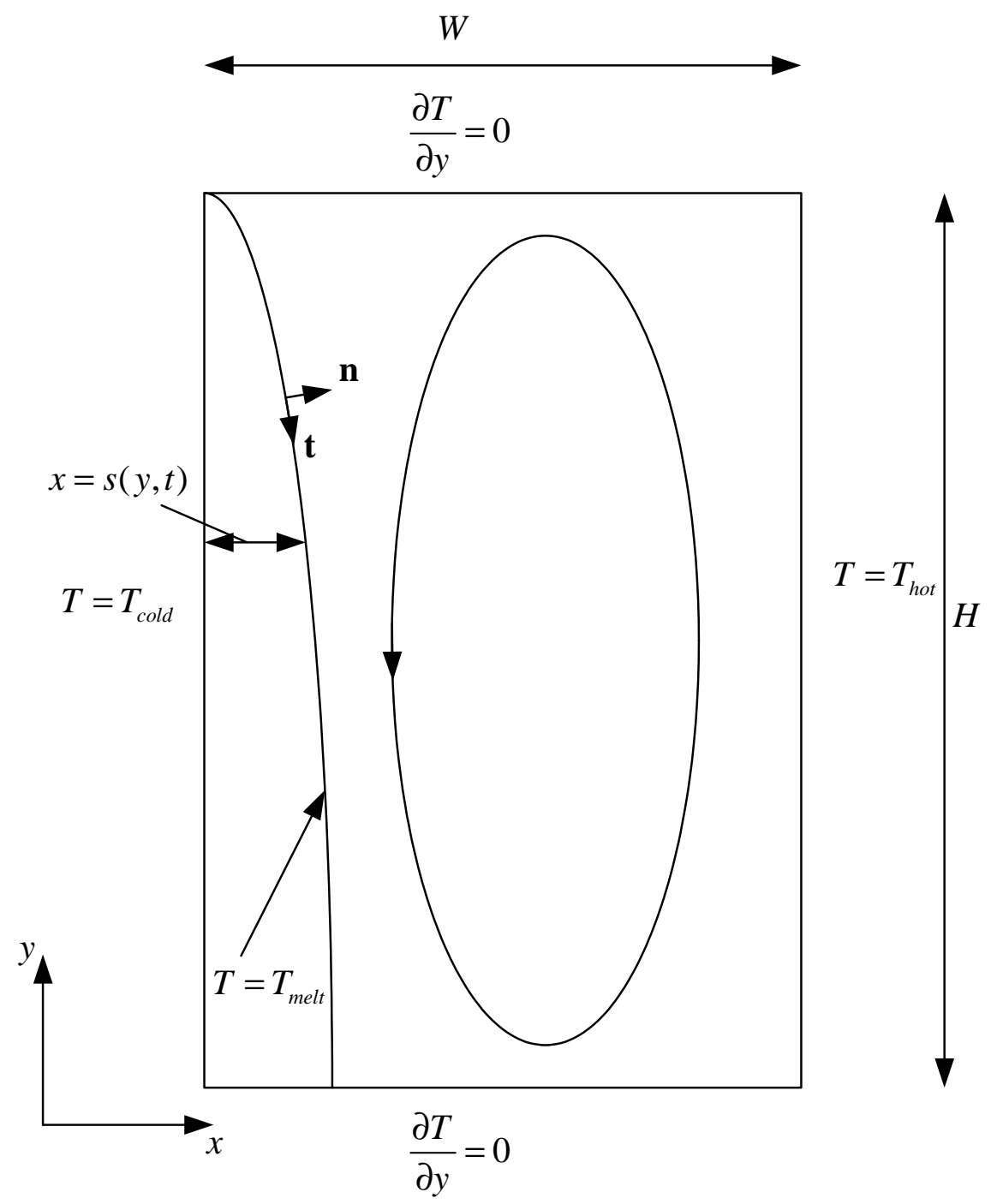

Fig. 1: Schematic for the freezing of water 


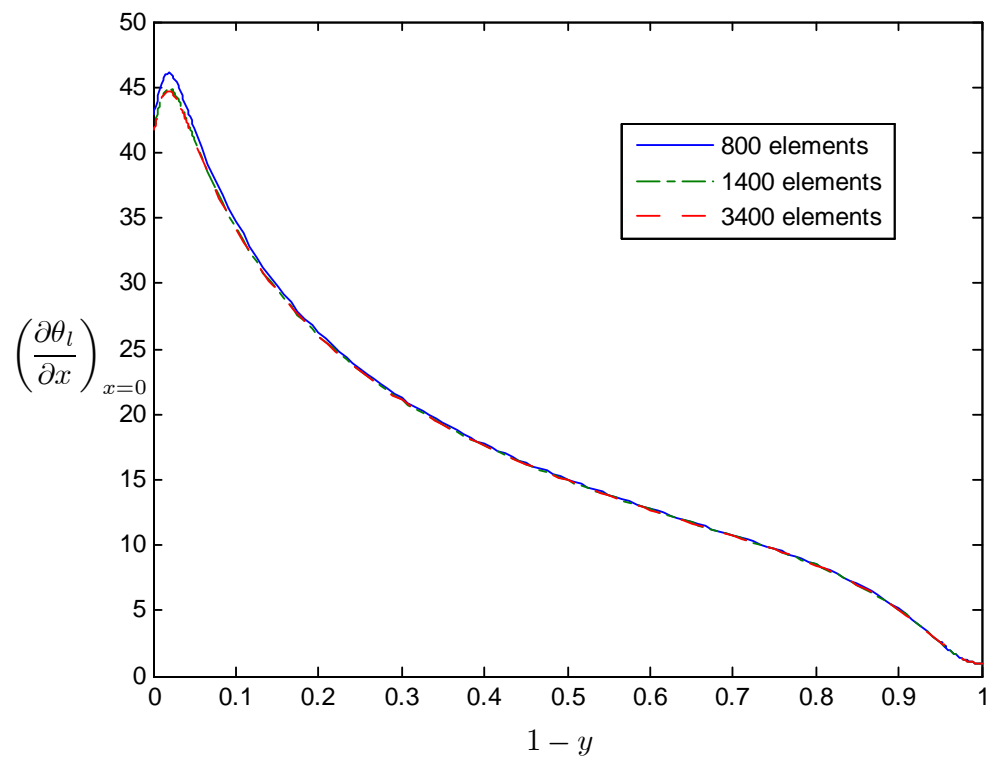

Fig. 2: Mesh independence study

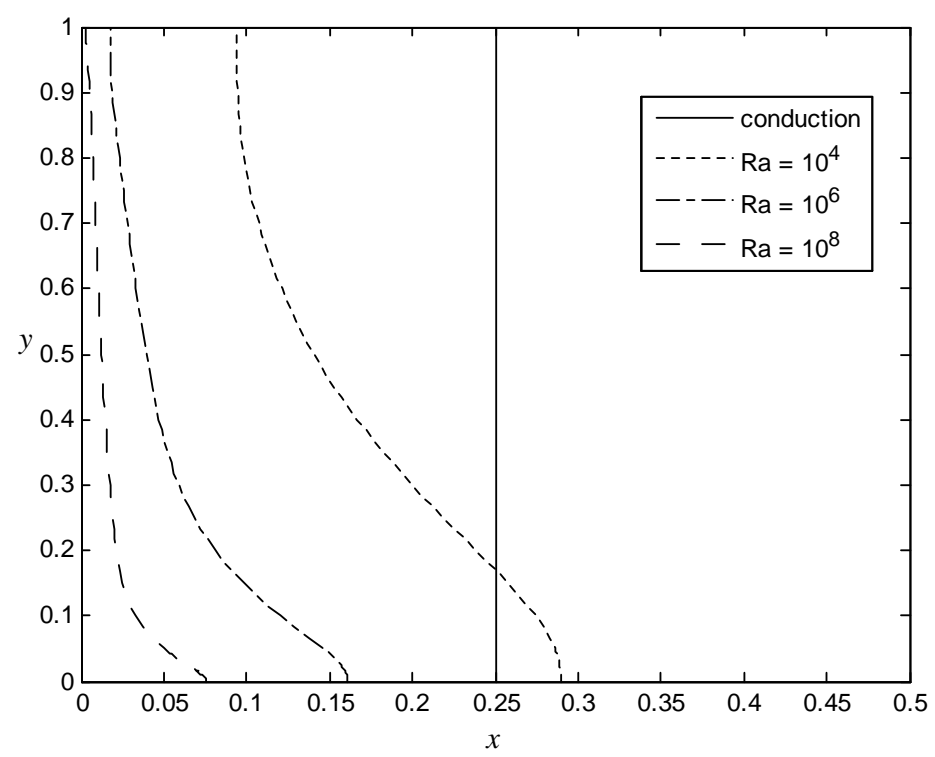

Fig. 3: Steady-state location of the solidification front for different values of $R a$ 


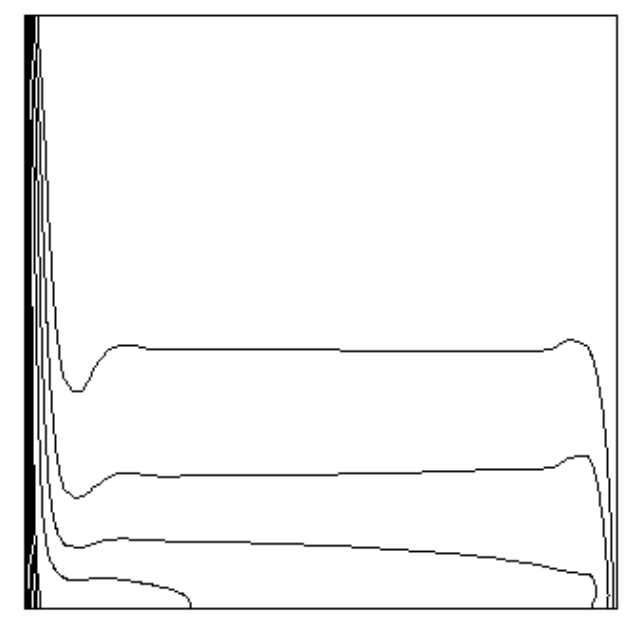

Fig. 4(a): Isotherms for flow at $R a=10^{7}$ at $\tau=10^{-2}$

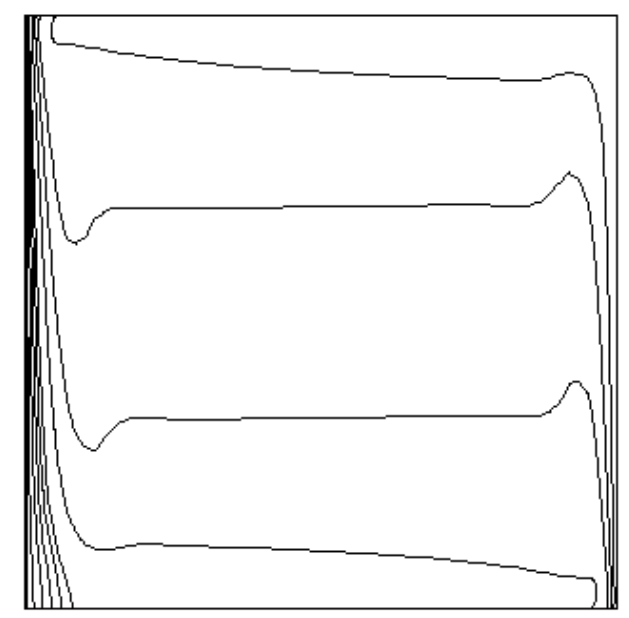

Fig. 4(b): Isotherms for flow at $R a=10^{7}$ at $\tau=10^{-1}$ 


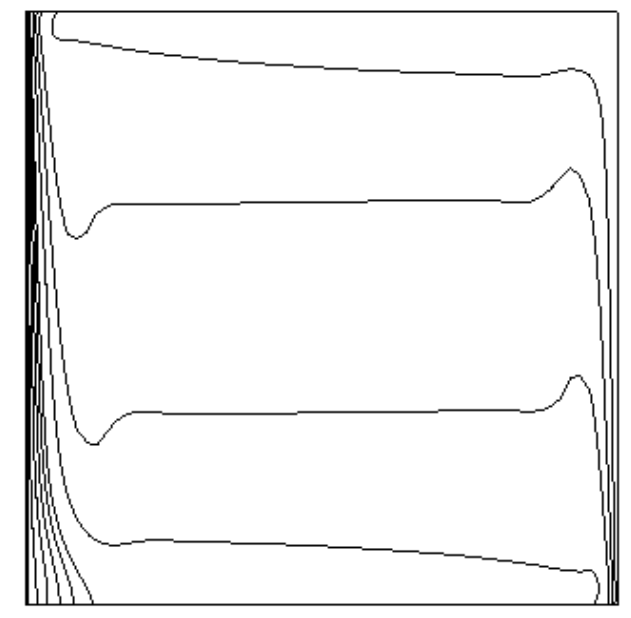

Fig. 4(c): Isotherms for flow at $R a=10^{7}$ at $\tau=1$ 


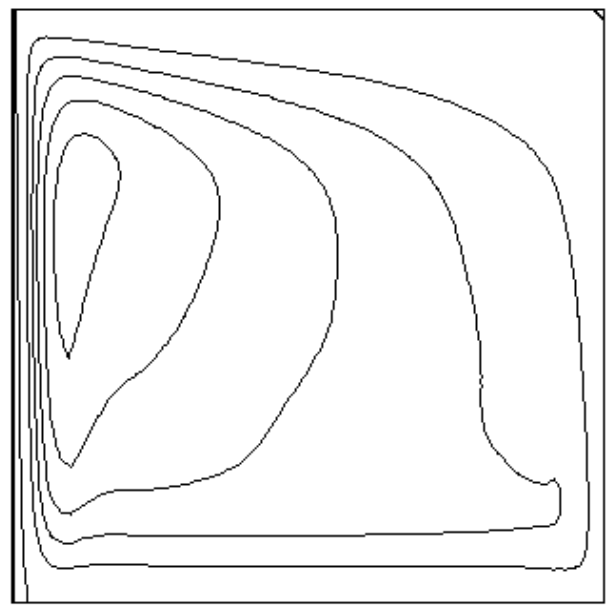

Fig. 5(a): Streamlines for flow at $R a=10^{7}$ at $\tau=10^{-2}$ $(\Delta \psi=10$, with $0 \leq \psi \leq 50)$

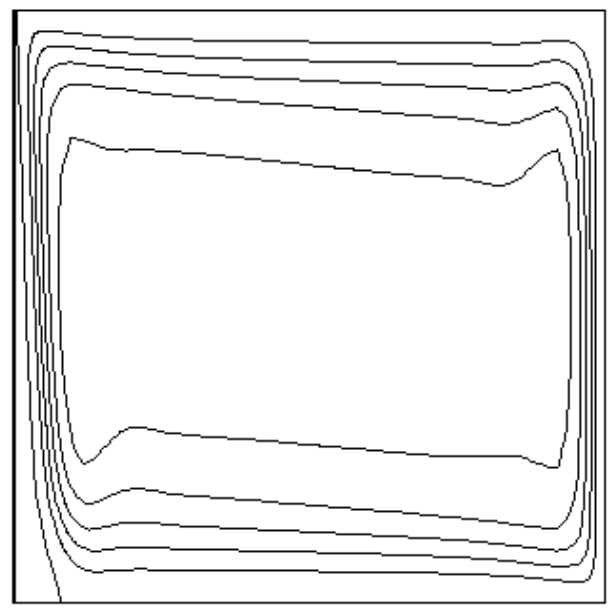

Fig. 5(b): Streamlines for flow at $R a=10^{7}$ at $\tau=10^{-1}$ $(\Delta \psi=6$, with $0 \leq \psi \leq 30)$ 


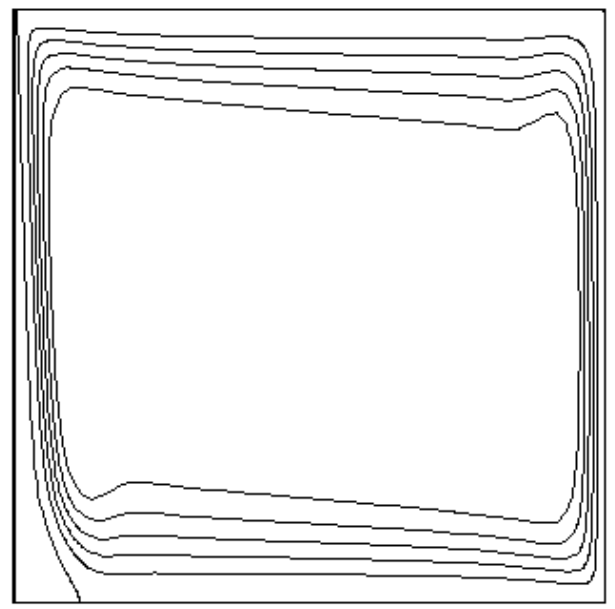

Fig. 5(c): Streamlines for flow at $R a=10^{7}$ at $\tau=1$ $(\Delta \psi=5$, with $0 \leq \psi \leq 25)$ 


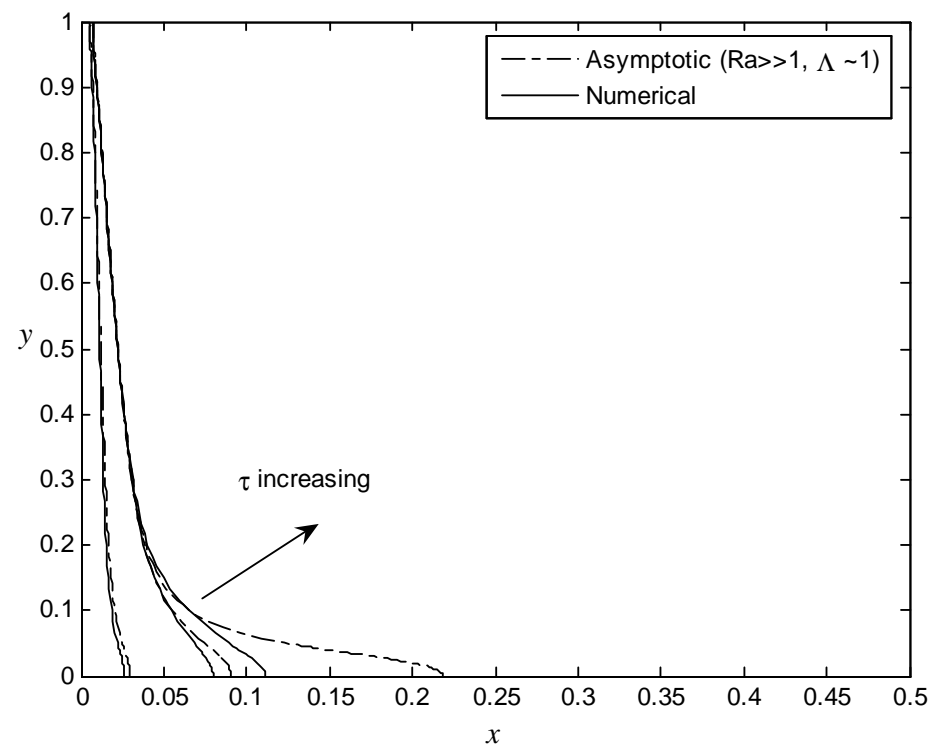

Fig. 6: Evolution of $s$ towards a steady state for $R a=10^{7}$, calculated using equation (39) and full numerical simulation, at $\tau=10^{-2}, 10^{-1}, 1$

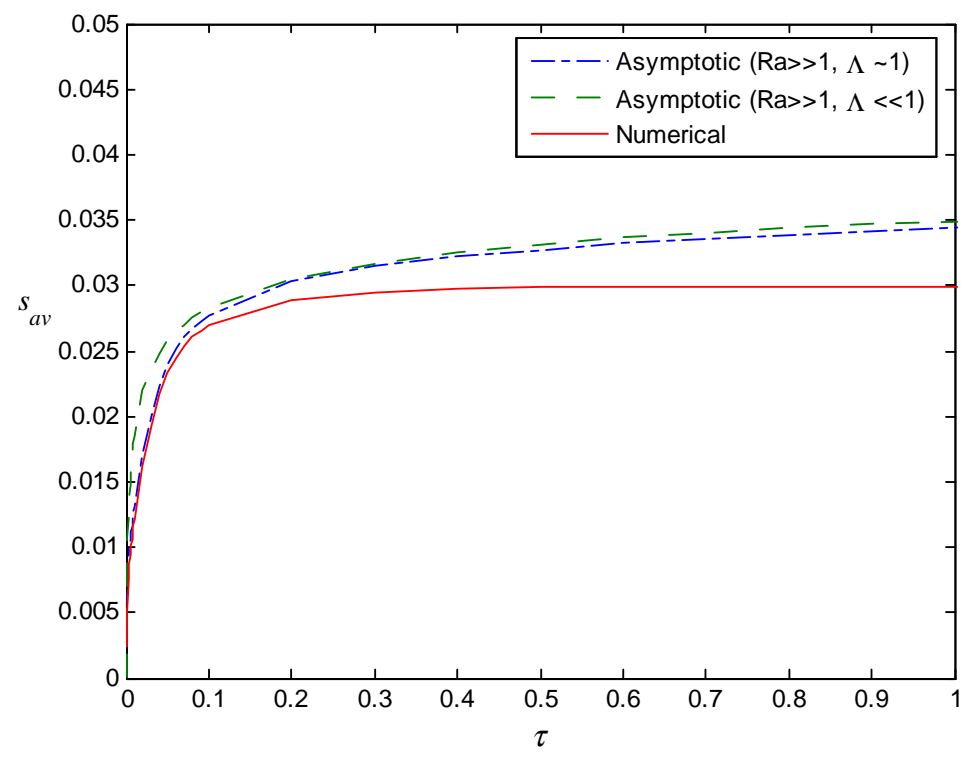

Fig. 7: Comparison of the average solid thickness, $s_{a v}$, as a function of $\tau$ for $R a=10^{7}$ 


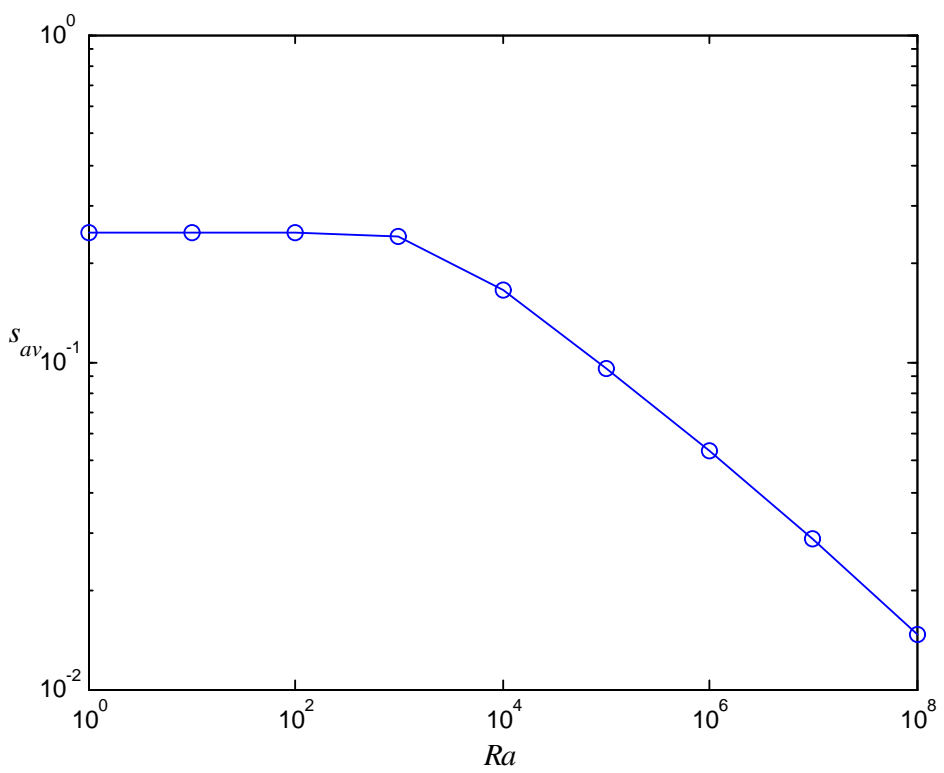

Fig. 8: $\log s_{a v}$ as a function of $\log R a$ 


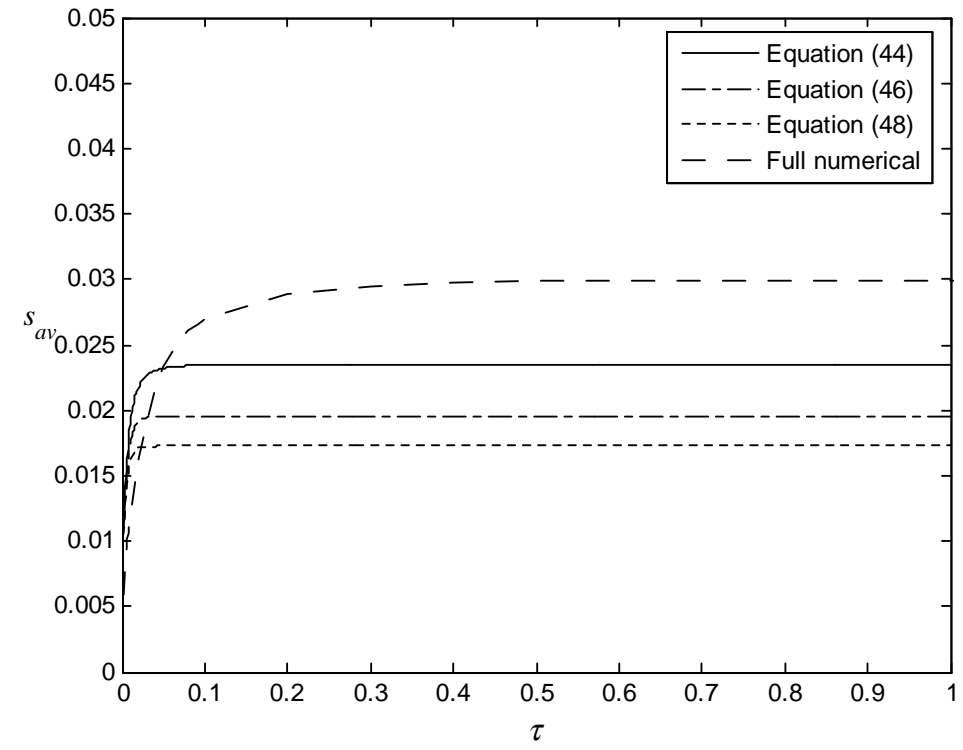

Fig. 9: Comparison of the time evolution of $s_{a v}$ for $R a=10^{7}$, using the full numerical solution and three different liquid phase-heat flux correlations 


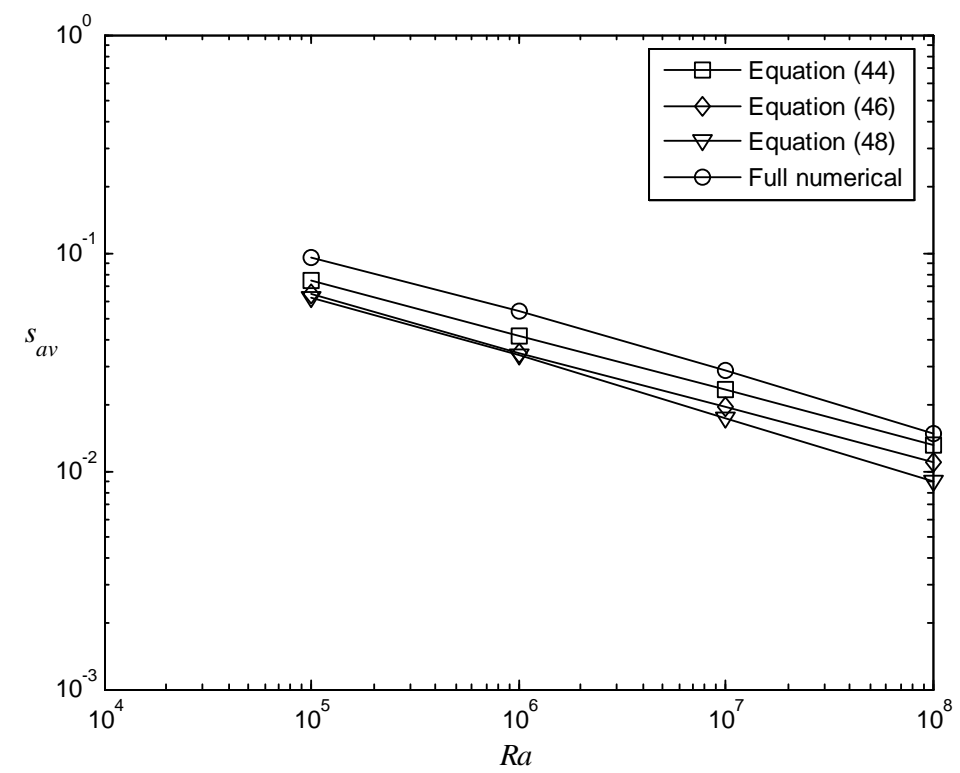

Fig. 10: Comparison of $\log \mathrm{s}_{a v}$ as a function of $\log R a$, using the full numerical solution and three different liquid-phase heat flux correlations 